\title{
Extended Poisson-Pareto Type II Distribution: Theoretical and Computational Aspects
}

Rania H. M. Abdelkhalek

Department of Statistics, Mathematics and Insurance,

Benha University, Egypt.

rania.abdelkhalek@fcom.bu.edu.eg

\begin{abstract}
We introduce a new continuous model with strong physical motivations and wide applications upon compounding the discrete zero truncated Poisson model and a new continuous model called the Burr X Pareto type II distribution. Some of its mathematical and statistical properties are derived as well as four applications to real data sets are provided with details to illustrate the wide importance of the new model. We conclude that the new model is better than other nine competitive models via the four applications. Method of maximum likelihood is used to estimate the unknown parameters of the new model. The new model provides adequate fits as compared to other related models in the four applications.
\end{abstract}

Keywords: Burr XII Distribution; Maximum Likelihood; Generating Function; Moments; Zero Truncated Poisson.

\section{Introduction and physical motivation}

A random variable (rv) $\mathrm{X}$ is said to have the one parameter Pareto type II (PaII) model if its probability density function (PDF) given by

$$
g_{\mathrm{PaII}}^{(\beta)}(x)=\frac{\beta}{(1+x)^{1+\beta}},
$$

and cumulative distribution function (CDF)

$$
G_{\text {PaII }}^{(\beta)}(x)=1-\frac{1}{(1+x)^{\beta}}
$$

where $\beta$ is a shape parameter. The PDF in (1) is a secial case from Burr type XII (BXII) model when $\alpha=1$

$$
g_{\mathrm{BXII}}^{(\alpha, \beta)}(x)=\alpha \beta x^{\alpha-1}\left(1+x^{\alpha}\right)^{-\beta-1}
$$

Due to Yousof et al. (2017a), we derive a new model called the Burr X PaII (BXPaII) model defined by the CDF given by

$$
H_{\mathrm{BXPaII}}^{(\theta, \beta)}(x)=\left(1-\exp \left\{-\left[(1+x)^{\beta}-1\right]^{2}\right\}\right)^{\theta},
$$

where $\theta>0$ is a shape parameter. When $\theta=1$, we obtain the one parameter Rayleigh PaII (RPaII) model. Suppose that we have a system has $N$ subsystems functioning independently at a given time where $N$ has zero truncated Poisson (ZTP) distribution with parameter $\lambda$. The probability mass function (PMF) of $N$ is given by

$$
\left.p_{\mathrm{ZTP}}^{(\lambda)}(N=n)\right|_{(n=1,2, \ldots)}=\left[\exp (-\lambda) \lambda^{n}\right] /\{n ![-\exp (-\lambda)+1]\}
$$

Note that for ZTP r.v., the expected value $(E(N \mid \lambda)), E\left(N^{2} \mid \lambda\right)$ and the variance $\operatorname{Var}(N \mid \lambda)$ are, respectively, given by

$$
\begin{aligned}
& E(N \mid \lambda)=\lambda /[-\exp (-\lambda)+1] \\
& E\left(N^{2} \mid \lambda\right)=\frac{\lambda(1+\lambda)}{[-\exp (-\lambda)+1]}
\end{aligned}
$$

and 


$$
\operatorname{Var}(N \mid \lambda)=\frac{\lambda+\lambda^{2}}{[-\exp (-\lambda)+1]}-\frac{\lambda^{2}}{[-\exp (-\lambda)+1]^{2}}
$$

Suppose that the failure time of each subsystem has the BXPaII. If $Y_{i}$ represents the failure time of the $i^{\text {th }}$ subsystem and let

$$
X=\min \left\{Y_{1}, Y_{2}, \cdots, Y_{N}\right\}
$$

Then the conditional CDF of $X \mid N$ is

$$
F(x \mid N)=1-\operatorname{Pr}(X>x \mid N)=1-\left[1-H_{\mathrm{BXPaII}}^{(\theta, \beta)}(x)\right]^{N} .
$$

Therefore, the unconditional CDF of the PBXPaII CDF can be expressed as

with the corresponding PDF as

$$
\left.F_{\mathrm{PBXPaII}}^{(\lambda, \theta, \beta)}(x)\right|_{\lambda \in R}=\frac{1-\exp \left[-\lambda\left(1-\exp \left\{-\left[(1+x)^{\beta}-1\right]^{2}\right\}\right)^{\theta}\right]}{-\exp (-\lambda)+1},
$$

$$
\begin{aligned}
& \left.f_{\mathrm{PBXPaII}}^{(\lambda, \theta, \beta)}(x)\right|_{\lambda \in R}=\frac{2 \theta \lambda \beta}{-\exp (-\lambda)+1}(1+x)^{2 \beta-1}\left[1-(1+x)^{-\beta}\right] \\
& \times \exp \left[-\lambda\left(1-\exp \left\{-\left[(1+x)^{\beta}-1\right]^{2}\right\}\right)^{\theta}\right] \\
\times & \exp \left\{-\left[(1+x)^{\beta}-1\right]^{2}\right\}\left(1-\exp \left\{-\left[(1+x)^{\beta}-1\right]^{2}\right\}\right)^{\theta-1} .
\end{aligned}
$$

The hazard rate function (HRF) can be easily calculated from $f_{\mathrm{PBXPaII}}^{(\lambda, \theta) /,(x)} /[1-$ $\left.F_{\mathrm{PBXPaII}}^{(\lambda, \theta, \beta)}(x)\right]$. Below some plots of the PDF and HRF for the new PBXPaII model.
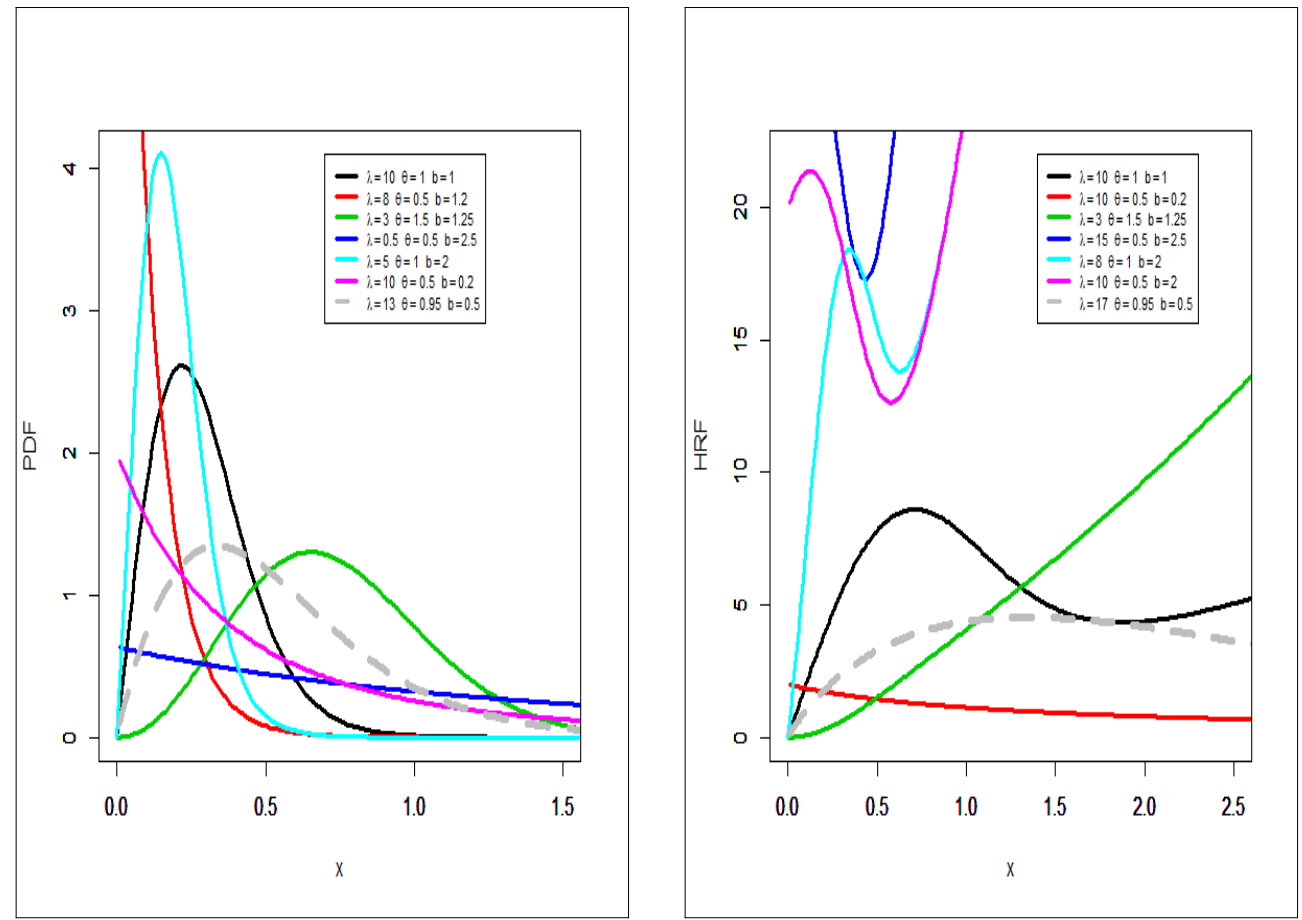

Figure 1: PDFs and HRFs plots for the PBXPaII model. 
The PBXPaII density can be right-skewed or unimodal whereas the HRF of the PBXPaII model can be unimodal or unimodal then bathtub or bathtub or increasing or unimodal then increasing (see Figure 1).

\section{Mathematical properties Useful expansions}

Using the power series

the PDF in (6) can be written as

$$
\sum_{m=0}^{\infty} \tau^{m} / m !=\exp (\tau)
$$

$$
\begin{aligned}
& \left.f_{\mathrm{PBXPaII}}^{(\lambda, \theta, \beta)}(x)\right|_{\lambda \in R}=\sum_{m=0}^{\infty} \frac{2 \theta \lambda^{1+m} a \beta(-1)^{m}}{m ![-\exp (-\lambda)+1]} \frac{\exp \left\{-\left[(1+x)^{\beta}-1\right]^{2}\right\}}{\left[1-(1+x)^{-\beta}\right]} \\
& \times(1+x)^{2 \beta-1}\left(1-\exp \left\{-\left[(1+x)^{\beta}-1\right]^{2}\right\}\right)^{(m+1) \theta-1}
\end{aligned}
$$

Considering the following power series holds

$$
\left.(1-s)^{c-1}\right|_{(|s|<1 \text { and } c>0 \text { is a realnon-integer })}=\sum_{i=0}^{\infty}\left\{\left[(-s)^{i} \Gamma(c)\right] /[i ! \Gamma(c-i)]\right\} .
$$

Upon applying (8) to (7) we have

$$
\begin{aligned}
& \left.f_{\mathrm{PBXPaII}}^{(\lambda, \theta, \beta)}(x)\right|_{\lambda \in R}=\frac{2 \theta a \beta(1+x)^{2 \beta-1}}{-\exp (-\lambda)+1}\left[1-(1+x)^{-\beta}\right] \\
& \times \sum_{m, i=0}^{\infty} \frac{\lambda^{1+m}(-1)^{m+i} \Gamma((m+1) \theta)}{i ! \Gamma((m+1) \theta-i)} \\
& \times \exp \left\{-(i+1)\left[(1+x)^{\beta}-1\right]^{2}\right\} .
\end{aligned}
$$

Via applying the power series to the term

then, equation (9) becomes

$$
\exp \left\{-(i+1)\left[(1+x)^{\beta}-1\right]^{2}\right\}
$$

$$
\begin{aligned}
\left.f_{\mathrm{PBXPaII}}^{(\lambda, \theta, \beta)}(x)\right|_{\lambda \in R}= & \sum_{m, i, w=0}^{\infty} \frac{2 \theta \lambda^{1+m} a \beta(-1)^{m+i+w}(i+1)^{w} \Gamma((m+1) \theta)}{i ! w ![-\exp (-\lambda)+1] \Gamma((m+1) \theta-i)} \\
& \times(1+x)^{-\beta-1} \frac{\left[1-(1+x)^{-\beta}\right]^{2 w+1}}{(1+x)^{-\beta(2 w+3)}}
\end{aligned}
$$

then, Equation (10) becomes

$$
\begin{aligned}
\left.f_{\mathrm{PBXPaII}}^{(\lambda, \theta, \beta)}(x)\right|_{\lambda \in R}= & \sum_{\substack{m, i, w, k=0\\
}}^{\infty} \frac{2 \theta \lambda^{1+m}(-1)^{m+i+w}(i+1)^{w}}{i ! w ! k ![-\exp (-\lambda)+1]} \frac{\Gamma((m+1) \theta) \Gamma(3+2 w+k)}{\Gamma((m+1) \theta-i) \Gamma(2 w+3)} \\
& \times \beta(1+x)^{-\beta-1}\left[1-(1+x)^{-\beta}\right]^{2 w+k+1} .
\end{aligned}
$$

Applying (8) to (11), Equation (11) becomes

where

$$
\left.f_{\mathrm{PBXPaII}}^{(\lambda, \theta, \beta)}(x)\right|_{\lambda \in R}=\sum_{r=0}^{\infty} c_{r} g_{\mathrm{PaII}}^{[(1+r) \beta]}(x),
$$




$$
\begin{aligned}
& c_{r}=\frac{(-1)^{r}}{\Gamma(2 w+k+2-r)(1+r) !} \\
& \times \sum \frac{2 \theta \lambda^{1+m}(-1)^{w} \Gamma(3+2 w+k) \Gamma(2 w+k+2)}{w ! k ![-\exp (-\lambda)+1] \Gamma(2 w+3)} \\
& \times \sum \frac{(-1)^{m+i} \Gamma((m+1) \theta)(i+1)^{w}}{i ! \Gamma((m+1) \theta-i)} \\
& m, i=0
\end{aligned}
$$

and $g_{\mathrm{PaII}}^{[(1+r) \beta]}(x)$ is the PaII density with parameter $(1+r) \beta$. Similarly, the CDF of the $\mathrm{PBXPaII}$ can also be expressed as

$$
\left.F_{\mathrm{PBXPaII}}^{(\lambda, \theta, \beta)}(x)\right|_{\lambda \in R}=\sum_{r=0}^{\infty} c_{r} G_{\mathrm{PaII}}^{[(1+r) \beta]}(x),
$$

where $G_{\mathrm{PaII}}^{[(1+r) \beta]}(x)$ is the PaII CDF with parameter $(1+r) \beta$.

\section{Quantile and random number generation}

The quantile function $(\mathrm{QF})$ of $X$, where $X \sim \operatorname{PBXPaII}(\lambda, \theta, \beta)$, is obtained by inverting (5) as

$$
Q(u)=\left[1-\left(1+\left\{-\ln \left[1-\left(\frac{-\ln \{1-u[-\exp (-\lambda)+1]\}}{\lambda}\right)^{\frac{1}{\theta}}\right]\right\}^{\frac{1}{2}}\right]^{\frac{1}{\beta}}-1\right.
$$

Simulating the PBXPaII r.v. is straightforward. If $U$ is a uniform variate on the unit intrval $(0,1)$, then the r.v. $X=Q(U)$ follows (5).

\section{The quantile spread (QS)}

The QS of rv $U \sim \operatorname{PBXPaII}(\lambda, \theta, \beta)$ is given by

and this implies

$$
\left.Q S_{U}(p)\right|_{(p \in(0.5,1))}=\left[F^{-1}(p)\right]-\left[F^{-1}(1-p)\right]
$$

where

$$
Q S_{U}(p)=\left[S^{-1}(1-p)\right]-\left[S^{-1}(p)\right]
$$

$$
F^{-1}(p)=S^{-1}(1-p) \text { and } S=1-F
$$

is the survival function. The QS of a distribution describes how the mass of probability is placed symmetrically about its median $\left(\operatorname{Med}\left(U_{i}\right)\right)$ and hence can be used to formalize concepts as tailweight traditionally associated with kurtosis. This way allows us to separate concepts like kurtosis ( $k u r\left(U_{i}\right)$ ) for asymmetric models. Let $U_{1}$ and $U_{2}$ be two rvs follow PBXPaII model with quantile spreads $Q S_{U_{1}}$ and $Q S_{U_{2}}$, respectively. Then $U_{1}$ is called smaller than or equal $U_{2}$ in quantile spread order, denoted as $U_{1} \leq_{Q S} U_{2}$, if

$$
Q S_{U_{1}}(p) \leq Q S_{U_{2}}(p), \forall p \in(0.5,1) \text {. }
$$

Below are some properties of the QS order

1 - The order $\leq_{Q S}$ is location-free

$$
U_{1} \leq_{Q S} U_{2} \text { if }\left(U_{1}+c\right) \leq\left._{Q S} U_{2}\right|_{(c \in R)} .
$$

$2-$ The order $\leq_{Q S}$ is dilative 


$$
U_{1} \leq_{Q S} a U_{1} \text { whenever } a \geq 1 \text { and } U_{2} \leq\left._{Q S} a U_{2}\right|_{(a \geq 1)} .
$$

3 - Assume $F_{U_{1}}$ and $F_{U_{2}}$ are symmetric, then

$$
U_{1} \leq_{Q S} U_{2} \text { if, and only if } F_{U_{1}}^{-1}(p) \leq\left._{Q S} F_{U_{2}}^{-1}(p)\right|_{(p \in(0.5,1))} .
$$

$4-$ The order $\leq_{Q S}$ implies ordering of the mean absolute deviation around the median, $\left.M A D\left(U_{i}\right)\right|_{i=1,2}$,

and

$$
\operatorname{MAD}\left(U_{1}\right)=E\left[\left|U_{1}-\operatorname{Med}\left(U_{1}\right)\right|\right]
$$

i.e.,

$$
\operatorname{MAD}\left(U_{2}\right)=E\left[\left|U_{2}-\operatorname{Med}\left(U_{2}\right)\right|\right],
$$

- Finally

$$
U_{1} \leq_{Q S} U_{2} \text { implies } M A D\left(U_{1}\right) \leq_{Q S} M A D\left(U_{2}\right)
$$

$$
U_{1} \leq_{Q S} U_{2} \text { iff }-U_{1} \leq_{Q S}-U_{2}
$$

\section{Moments}

The $r^{\text {th }}$ ordinary moment of $X$, say $\mu_{r}^{\prime}$, follows from (12) as

$$
\mu_{n}^{\prime}=E\left(X^{n}\right)=\left.\sum_{r=0}^{\infty} \quad c_{r}(1+r) \beta B((1+r) \beta-n, 1+n)\right|_{[n<(1+r) \beta]} .
$$

Setting $n=1$ in (13) gives the mean of $X$. The $n^{\text {th }}$ incomplete moment of $X$ is defined by

We can write from (12)

$$
\tau_{n}(t)=\int_{-\infty}^{t} x^{r} f(x) d x
$$

$$
\tau_{n}(t)=\left.\sum_{r=0}^{\infty} c_{r}(1+r) \beta B\left(t^{a} ;(1+r) \beta-n, 1+n\right)\right|_{[n<(1+r) \beta]},
$$

where

$$
B(a, \beta)=\int_{0}^{\infty} x^{a-1}(1+x)^{-(a+\beta)} d x
$$

and

$$
B(t ; a, \beta)=\int_{0}^{t} x^{a-1}(1+x)^{-(a+\beta)} d x
$$

are the beta and the incomplete beta functions of the second type, respectively. Two important applications of the first incomplete moment $\left(\tau_{n=1}(t)\right)$ are related to the mean deviations about the mean (MD (mean) ), mean deviations about the median (MD (median) ), the Bonferroni and Lorenz curves.

\section{Residual and reversed residual life functions}

The $n^{\text {th }}$ moment of the residual life (MRL) $\left[m_{n}(t)\right]$, denoted by

$$
m_{n}(t)=E\left[\left.(X-t)^{n}\right|_{(X>t, n=1,2, \ldots)}\right],
$$

which uniquely determine $F(x)$. The $n^{\text {th }}$ MRL of $X$ is given by

so, we can write

$$
m_{n}(t)=\frac{\int_{t}^{\infty}(x-t)^{n} d F(x)}{1-F(t)}
$$




$$
\begin{gathered}
m_{n}(t)=\frac{1}{1-F(t)} \sum_{r=0}^{\infty} \sum_{i=0}^{n} \frac{(-1)^{n-i} n ! t^{n-i}}{i ! \Gamma(n-i+1)} \\
\times\left. c_{r}(1+r) \beta B(t,(1+r) \beta-n, 1+n)\right|_{[n<(1+r) \beta]},
\end{gathered}
$$

setting $n=1$, we get the mean residual life (MRL) function or the life expectation at age $x$ which defined by

$$
m_{1}(x)=E\left[\left.(X-x)\right|_{(X>t, n=1)}\right]
$$

which represents the expected additional life length for a unit which is alive at age $x$.

The $n^{\text {th }}$ moment of the reversed residual life (MRRL) $\left[M_{n}(t)\right]$, denoted by

$$
M_{n}(t)=E\left[\left.(t-X)^{n}\right|_{(X \leq t, t>0 \text { and } n=1,2, \ldots)}\right],
$$

which also uniquely determines $F(x)$. Then, the $M_{n}(t)$ can be formulated as

$$
M_{n}(t)=\frac{\int_{0}^{t}(t-x)^{n} d F(x)}{F(t)}
$$

so that, the $n^{\text {th }}$ moment of the reversed residual life of $X$

$$
M_{n}(t)=\left.\frac{1}{F(t)} \sum_{i=0}^{n} \sum_{r=0}^{\infty} \frac{(-1)^{i} n !}{i !(n-i) !} c_{r}(1+r) \beta B(t,(1+r) \beta-n, 1+n)\right|_{[n<(1+r) \beta]},
$$

setting $n=1$ in the above equation, we get the mean inactivity time or mean waiting time which also called the mean reversed residual life function

$$
M_{n}(t)=E\left[\left.(t-X)^{n}\right|_{(X \leq t, t>0 \text { and } n=1)}\right]
$$

which represents the waiting time elapsed since the failure of an item on condition that this failure had occurred in $(0, x)$.

\section{Parameter estimation}

In this work, we will estimate the unknown parameters $(\lambda, \theta, \beta)$ of the PBXPaII model from the complete samples by maximum likelihood (ML) method. Suppose that $x_{1}, \cdots, x_{n}$ be a random sample from the PBXPaII model with parameter vector $\Phi=(\lambda, \theta, \beta)^{T}$. The log-likelihood function $\left(\ell_{n}(\Phi)\right)$ for $\Phi$ is given by

$$
\begin{aligned}
& \ell_{n}(\Phi)=n \log 2+n \log \theta+n \log \lambda+n \log \beta-n \log [-\exp (-\lambda)+1] \\
& +(a-1) \sum_{i=1}^{n} \log x_{i}+(2 \beta-1) \sum_{i=1}^{n} \log s_{i}+\sum_{i=1}^{n} \log \left(1-s_{i}^{-\beta}\right) \\
& -\lambda \sum_{i=1}^{n}\left(1-z_{i}\right)^{\theta}+\sum_{i=1}^{n} \log z_{i}+(\theta-1) \sum_{i=1}^{\log \left(1-z_{i}\right)}
\end{aligned}
$$

where

$$
s_{i}=1+x_{i} \text { and } z_{i}=\exp \left[-\left(-1+s_{i}^{\beta}\right)^{2}\right]
$$

The above $\ell_{n}(\Phi)$ can be maximized numerically via SAS (PROC NLMIXED) or R (optim) or Ox program (via sub-routine MaxBFGS), among others. The components of the score vector

are easily to be derived.

$$
U(\Theta)=\frac{\partial \ell}{\partial \Theta}=\left(\frac{\partial \ell_{n}(\Phi)}{\partial \lambda}, \frac{\partial \ell_{n}(\Phi)}{\partial \theta}, \frac{\partial \ell_{n}(\Phi)}{\partial \beta}\right)^{T}
$$




\section{Applications}

Four applications are provided to illustrate the potentiality, importance and flexibility of the PBXPaII model. For all data sets, we compare the PBXPaII distribution with other nine extension of the BXII such as Marshall-Olkin BXII (MOBXII), Zografos-Balakrishnan BXII (ZBBXII), BBXII, Beta exponentiated BXII (BEBXII), Topp Leone BXII (TLBXII), five Parameters BBXII (FBBXII), five Parameters Kumaraswamy BXII (FKwBXII), KwBXII and the BXII distributions given in Yousof et al. (2017b), Altun et al. (2018a, b), Yousof et al. (2018a, b), Korkmaz et al. (2018a, b), Alizadeh et al. (2018), Ibrahim (2019), Korkmaz et al. (2019), Hamedani et al. (2019), Nascimento et al. (2019), Alizadeh et al. (2019) and Yousof et al. (2019).

Data Set I $\{0.98,5.56,5.08,0.39,1.57,3.19,4.90,2.93,2.85,2.77,2.76,1.73,2.48,3.68$, $1.08,3.22,3.75,3.22,3.70,2.74,2.73,2.50,3.60,3.11,3.27,2.87,1.47,3.11,4.42,2.40$, $3.15,2.67,3.31,2.81,2.56,2.17,4.91,1.59,1.18,2.48,2.03,1.69,2.43,3.39,3.56,2.83$, $3.68,2.00,3.51,0.85,1.61,3.28,2.95,2.81,3.15,1.92,1.84,1.22,2.17,1.61,2.12,3.09$, 2.97, 4.20, 2.35, 1.41, 1.59, 1.12, 1.69, 2.79, 1.89, 1.87, 3.39, 3.33, 2.55, 3.68, 3.19, 1.71, $1.25,4.70,2.88,2.96,2.55,2.59,2.97,1.57,2.17,4.38,2.03,2.82,2.53,3.31,2.38,1.36$, $0.81,1.17,1.84,1.80,2.05,3.65\}$ called breaking stress data. This data set consists of 100 observations of breaking stress of carbon fibers (in Gba) given by Nichols and Padgett (2006).

Data Set II $\{0.1,0.33,0.44,0.56,0.59,0.72,0.74,0.77,0.92,0.93,0.96,1,1,1.02,1.05$, 1.07, 07, 1.08, 1.08, 1.08, 1.09, 1.12, 1.13, 1.15, 1.16, 1.2, 1.21, 1.22, 1.22, 1.24, 1.3, 1.34, $1.36,1.39,1.44,1.46,1.53,1.59,1.6,1.63,1.63,1.68,1.71,1.72,1.76,1.83,1.95,1.96$, $1.97,2.02,2.13,2.15,2.16,2.22,2.3,2.31,2.4,2.45,2.51,2.53,2.54,2.54,2.78,2.93$, $3.27,3.42,3.47,3.61,4.02,4.32,4.58,5.55\}$ called survival times in days of 72 guinea pigs infected with virulent tubercle bacilli, originally observed and reported by Bjerkedal (1960).

Data Set III $\{5.9,20.4,14.9,16.2,17.2,7.8,6.1,9.2,10.2,9.6,13.3,8.5,21.6,18.5,5.1,6.7$, $17,8.6,9.7,39.2,35.7,15.7,9.7,10,4.1,36,8.5,8,9.2,26.2,21.9,16.7,21.3,35.4,14.3$, $8.5,10.6,19.1,20.5,7.1,7.7,18.1,16.5,11.9,7,8.6,12.5,10.3,11.2,6.1,8.4,11,11.6$, $11.9,5.2,6.8,8.9,7.1,10.8\}$ called taxes revenue data. The actual taxes revenue data (in 1000 million Egyptian pounds) (see Altun et al. (2018a, b)).

Data set IV $\{65,156,100,134,16,108,121,4,39,143,56,26,22,1,1,5,65,56,65,17$, $7,16,22,3,4,2,3,8,4,3,30,4,43\}$ called leukaemia data. This real data set gives the survival times, in weeks, of 33 patients suffering from acute Myelogeneous Leukaemia (see Altun et al. (2018a, b)).

We consider the following goodness-of-fit statistics: the Akaike information criterion (AIC), Bayesian information criterion (BIC), Hannan-Quinn information criterion (HQIC), consistent Akaike information criterion (CAIC). Generally, the smaller these statistics are, the better the fit. Based on the values in Tables 1-4 and Figure 2-7 the PBXPaII model 
provides the best fits as compared to other models in the four applications with small values for BIC, AIC, CAIC and HQIC.

Total time test (TTT) plot (see Figure 2) is an important graphical approach to verify whether our data can be applied to a specific model or not. Due to Aarset (1987), the empirical version of the TTT plot is given by plotting

$$
T\left(\frac{r}{n}\right)=\frac{\sum_{i=1}^{r} y_{i: n}+(n-r) y_{r}: n}{\sum_{i=1}^{n} y_{i: n}}
$$

against $r / n$, where $r=1,2, n$ and $y_{i}: n(i=1, ?, n)$ are the order statistics of the sample. Aarset (1987) showed that the HRF is constant if the TTT plot is graphically presented as a straight diagonal, the HRF is increasing (or decreasing) if the TTT plot is concave (or convex). The HRF is U-shaped (bathtub) if the TTT plot is firstly convex and then concave, if not, the HRF is unimodal. The TTT plots the four real data sets are presented in Figure 2. This plot indicates that the empirical HRFs of the the four data sets are decreasing, decreasing, decreasing and unimodal.

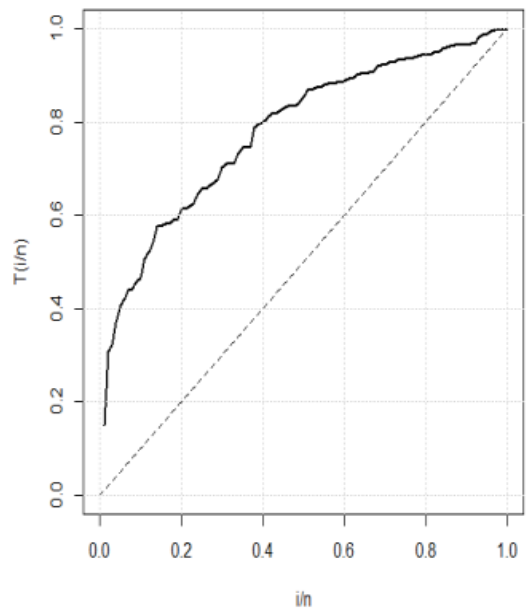

Data set I

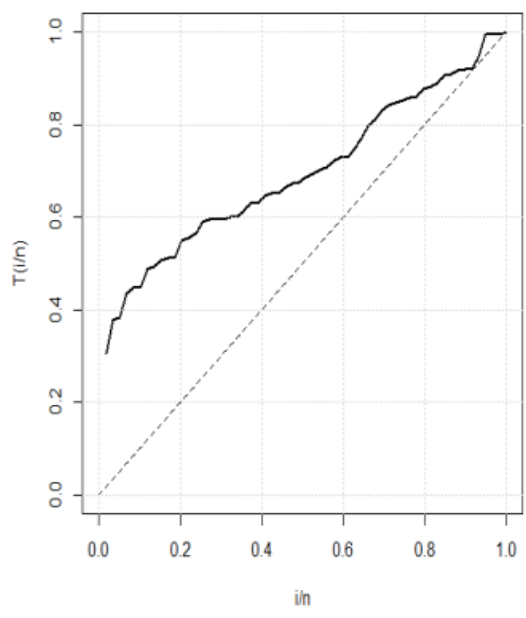

Data set III

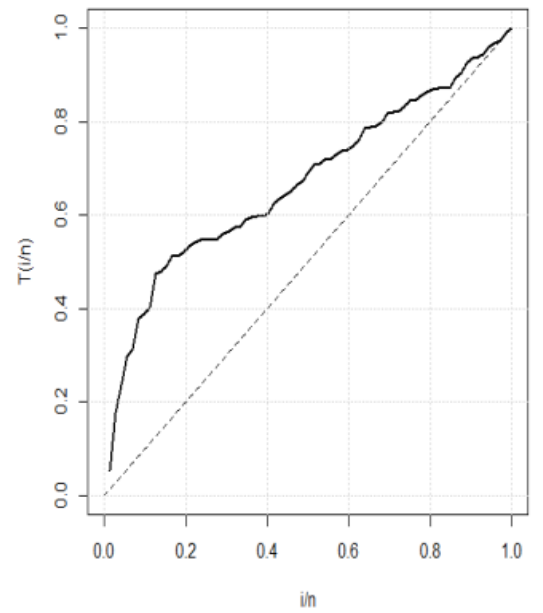

Data set II

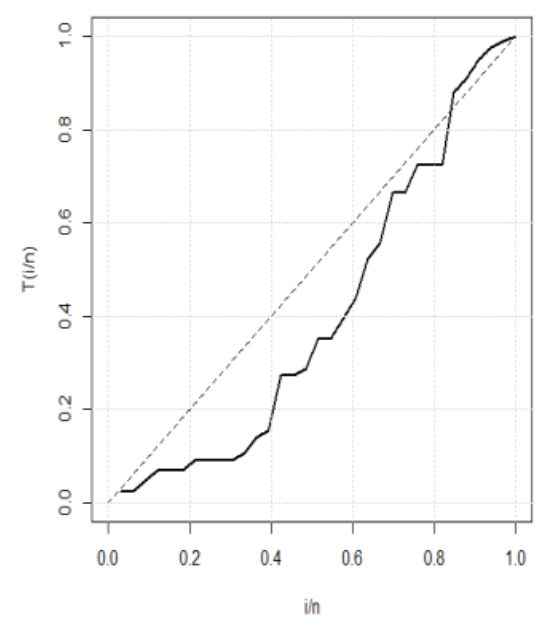

Data set IV

Figure 2: TTT plots. 
Table 1: MLEs and standard errors, confidence interval (in parentheses) with

AIC, BIC, CAIC and HQIC values for the data set I.

\begin{tabular}{|c|c|c|}
\hline Model & $\hat{\lambda}, \hat{\theta}, \hat{\alpha}, \hat{\beta}, \hat{\gamma}$ & AIC, BIC, CAIC, HQIC \\
\hline \multirow[t]{3}{*}{ BXII } & ---,---, 5.941, 0.187,--- & $382.94,388.15,383.06,385.05$ \\
\hline & $-------,(1.279),(0.044),---$ & \\
\hline & $---,---,(3.43,8.45),(0.10,0.27),---$ & \\
\hline \multirow[t]{3}{*}{ MOBXII } & $---,---, 1.192,4.834,838.73$ & $305.78,313.61,306.03,308.96$ \\
\hline & $---,---,(0.952),(4.896),(229.34)$ & \\
\hline & $---,---, 0,3.06),(0,14.43),(389.22,1288.24)$ & \\
\hline \multirow[t]{3}{*}{ TLBXII } & $---,---, 1.350,1.061,13.728$ & $323.52,331.35,323.77,326.70$ \\
\hline & $---,---, 0.378),(0.384),(8.400)$ & \\
\hline & $---,---,(0.61,2.09),(0.31,1.81),(0,30.19)$ & \\
\hline \multirow[t]{3}{*}{ KwBXII } & $48.103,79.516,0.351,2.730,---$ & $303.76,314.20,304.18,308.00$ \\
\hline & (19.348), (58.186), (0.098), (1.077),--- & \\
\hline & (10.18,86.03), (0,193.56), (0.16,0.54), (0.62,4.84),--- & \\
\hline \multirow[t]{3}{*}{ BBXII } & $359.683,260.097,0.175,1.123,---$ & $305.64,316.06,306.06,309.85$ \\
\hline & $(57.941),(132.213),(0.013),(0.243),---$ & \\
\hline & $(246.1,473.2),(0.96,519.2),(0.14,0.20),(0.65,1.6),---$ & \\
\hline \multirow[t]{3}{*}{ BEBXII } & $0.381,11.949,0.937,33.402,1.705$ & $305.82,318.84,306.46,311.09$ \\
\hline & $(0.078),(4.635),(0.267),(6.287),(0.478)$ & \\
\hline & $(0.23,0.53),(2.86,21),(0.41,1.5),(21,45),(0.8,2.6)$ & \\
\hline \multirow[t]{3}{*}{ FKwBXII } & $0.542,4.223,5.313,0.411,4.152$ & $305.50,318.55,306.14,310.80$ \\
\hline & $(0.137),(1.882),(2.318),(0.497),(1.995)$ & \\
\hline & $(0.3,0.8),(0.53,7.9),(0.9,9),(0,1.7),(0.2,8)$ & \\
\hline \multirow[t]{3}{*}{ ZBBXII } & $123.101,---, 0.368,139.247,---$ & $302.96,310.78,303.21,306.13$ \\
\hline & $(243.011),---,(0.343),(318.546),---$ & \\
\hline & $(0,599.40),---,(0,1.04),(0,763.59),---$ & \\
\hline \multirow[t]{3}{*}{ PBXPaII } & $-2.25,1.66,---, 0.67,---$ & 288.7, 296.5, 288.9, 291.8 \\
\hline & $(1.6),(0.78),---,(0.02),---$ & \\
\hline & $(-5.45,0.95),(0.06,3.3),---,(0.63,0.71),---$ & \\
\hline
\end{tabular}


Table 2: MLEs and standard errors, confidence interval (in parentheses) with AIC, BIC, CAIC and HQIC values for the data set II.

\begin{tabular}{|c|c|c|}
\hline Model & $\hat{\lambda}, \hat{\theta}, \hat{\alpha}, \hat{\beta}, \hat{\gamma}$ & AIC, BIC, CAIC, HQIC \\
\hline \multirow[t]{3}{*}{ BXII } & $---,---, 3.102,0.465,---$ & $209.60,214.15,209.77,211.40$ \\
\hline & $----,--,(0.538),(0.077),---$ & \\
\hline & $---,---,(2.05,4.16),(0.31,0.62),---$ & \\
\hline \multirow[t]{3}{*}{ MOBXII } & $---,---, 2.259,1.533,6.760$ & $209.74,216.56,210.09,212.44$ \\
\hline & $---,---,(0.864),(0.907),(4.587)$ & \\
\hline & $---,--,(0.57,3.95),(0,3.31),(0,15.75)$ & \\
\hline \multirow[t]{3}{*}{ TLBXII } & $---,---, 2.393,0.458,1.796$ & $211.80,218.63,212.15,214.52$ \\
\hline & $---,---,(0.907),(0.244),(0.915)$ & \\
\hline & $---,--,(0.62,4.17),(0,0.94),(0.002,3.59)$ & \\
\hline \multirow[t]{3}{*}{ TLBXII } & $---,---, 2.393,0.458,1.796$ & $211.80,218.63,212.15,214.52$ \\
\hline & $---,---,(0.907),(0.244),(0.915)$ & \\
\hline & $---,--,(0.62,4.17),(0,0.94),(0.002,3.59)$ & \\
\hline \multirow[t]{3}{*}{ KwBXII } & $14.105,7.424,0.525,2.274,---$ & $208.76,217.86,209.36,212.38$ \\
\hline & $(10.805),(11.850),(0.279),(0.990),---$ & \\
\hline & $(0,35.28),(0.30 .65),(0,1.07),(0.33,4.21),---$ & \\
\hline \multirow[t]{3}{*}{ FBBXII } & $0.621,0.549,3.838,1.381,1.665$ & $206.80,218.20,207.71,211.30$ \\
\hline & $(0.541),(1.011),(2.785),(2.312),(0.436)$ & \\
\hline & $(0,1.7),(0,2.5),(0,9.3),(0,5.9),(0.8,4.5)$ & \\
\hline \multirow[t]{3}{*}{ FKwBXII } & $0.558,0.308,3.999,2.131,1.475$ & $206.50,217.90,207.41,211.00$ \\
\hline & $(0.442),(0.314),(2.082),(1.833),(0.361)$ & \\
\hline & $(0,1.4),(0,0.9),(0,3.1),(0,5.7),(0.76,2.2)$ & \\
\hline \multirow[t]{3}{*}{ PBXPaII } & 2.6, 0.69, ---,0.238,--- & 203.7, 210.5, 204, 206.4 \\
\hline & $(1.56),(0.098),---,(0.043),---$ & \\
\hline & $(0,5.7),(0,2.5),---,(0.15,0.324),---$ & \\
\hline
\end{tabular}


Table 3: MLEs and standard errors, confidence interval (in parentheses) with AIC, BIC, CAIC and HQIC values for the data set III.

\begin{tabular}{|c|c|c|}
\hline Model & $\hat{\lambda}, \hat{\theta}, \hat{\alpha}, \hat{\beta}, \hat{\gamma}$ & AIC, BIC, CAIC, HQIC \\
\hline \multirow[t]{3}{*}{ BXII } & ----,--- 5.615, 0.072,--- & $518.46,522.62,518.67,520.08$ \\
\hline & $---,---,(15.048),(0.194),---$ & \\
\hline & $---,---,(0,35.11),(0,0.45),---$ & \\
\hline \multirow[t]{3}{*}{ MOBXII } & ---,---, 8.017, 0.419, 70.359 & $387.22,389.38,387.66,389.68$ \\
\hline & $---,---,(22.083),(0.312),(63.831)$ & \\
\hline & $---,---,(0,51.29),(0,1.03),(0,195.47)$ & \\
\hline \multirow[t]{3}{*}{ TLBXII } & $---,---, 91.320,0.012,141.073$ & $385.94,392.18,386.38,388.40$ \\
\hline & $---,---,(15.071),(0.002),(70.028)$ & \\
\hline &,,$------(61.78,120.86)(0.008,0.02)(3.82,278.33)$ & \\
\hline \multirow[t]{3}{*}{ KwBXII } & $18.130,6.857,10.694,0.081,---$ & $385.58,393.90,386.32,388.86$ \\
\hline & (3.689), (1.035), (1.166), $(0.012),---$ & \\
\hline & $(10.89,25.36),(4.83,8.89),(8.41,12.98),(0.06,0.10),---$ & \\
\hline \multirow[t]{3}{*}{ BBXII } & $26.725,9.756,27.364,0.020,---$ & $385.56,394.10,386.30,389.10$ \\
\hline & $(9.465),(2.781),(12.351),(0.007),---$ & \\
\hline & $(8.17,45.27),(4.31,15.21),(3.16,51.57),(0.006,0.03),---$ & \\
\hline \multirow[t]{3}{*}{ BEBXII } & $2.924,2.911,3.270,12.486,0.371$ & $387.04,397.42,388.17,391.09$ \\
\hline & $(0.564),(0.549),(1.251),(6.938),(0.788)$ & \\
\hline & $(1.82,4.03),(1.83,3.99),(0.82,5.72),(0,26.08),(0,1.92)$ & \\
\hline \multirow[t]{3}{*}{ FBBXII } & $30.441,0.584,1.089,5.166,7.862$ & $386.74,397.14,387.87,390.84$ \\
\hline & $(91.745),(1.064),(1.021),(8.268),(15.036)$ & \\
\hline & $(0,210.26),(0,2.67),(0,3.09),(0,21.37),(0,37.33)$ & \\
\hline \multirow[t]{3}{*}{ FKwBXII } & $12.878,1.225,1.665,1.411,3.732$ & $386.96,397.36,388.09,391.06$ \\
\hline & $(3.442),(0.131),(0.034),(0.088),(1.172)$ & \\
\hline & $(6.1,19.6),(0.9,1.48),(1.56,1.73),(1.24,1.58),(1.4,6.03)$ & \\
\hline \multirow[t]{3}{*}{ PBXPaII } & $1.78,4.78,---, 00.32,---$ & $384.5,390.7,384.9,386.9$ \\
\hline & (1.37), (0.92), ---,(0.025),--- & \\
\hline & $(0,4.5),(3,6.6),---,(0.27,0.37),---$ & \\
\hline
\end{tabular}


Table 4: MLEs and standard errors, confidence interval (in parentheses) with AIC, BIC, CAIC and HQIC values for the data set IV.

\begin{tabular}{|c|c|c|}
\hline Model & $\hat{\lambda}, \hat{\theta}, \hat{\alpha}, \hat{\beta}, \hat{\gamma}$ & AIC, BIC, CAIC, HQIC \\
\hline \multirow[t]{3}{*}{ BXII } & $---,---, 58.711,0.006,---$ & $328.20,331.19,328.60,329.19$ \\
\hline & $---,---,(42.382),(0.004),---$ & \\
\hline & $---,---,(0,141.78),(0,0.01),---$ & \\
\hline \multirow[t]{3}{*}{ MOBXII } & $---,---, 11.838,0.078,12.251$ & $315.54,320.01,316.37,317.04$ \\
\hline & $---,---,(4.368),(0.013),(7.770)$ & \\
\hline & $---,---,(0,141.78),(0,0.01),(0,27.48)$ & \\
\hline \multirow[t]{3}{*}{ TLBXII } & $---,---, 0.281,1.882,50.215$ & $316.26,320.73,317.09,317.76$ \\
\hline & $---,---,(0.288),(2.402),(176.50)$ & \\
\hline & $---,---,(0,0.85),(0,6.59),(0,396.16)$ & \\
\hline \multirow[t]{3}{*}{ KwBXII } & $9.201,36.428,0.242,0.941,---$ & $317.36,323.30,318.79,319.34$ \\
\hline & $(10.060),(35.650),(0.167),(1.045),---$ & \\
\hline & $(0,28.912),(0,106.30),(0,0.57),(0,2.99),---$ & \\
\hline \multirow[t]{3}{*}{ BBXII } & 96.104, 52.121, 0.104, 1.227,--- & $316.46,322.45,317.89,318.47$ \\
\hline & $(41.201),(33.490),(0.023),(0.326),---$ & \\
\hline & $(15.4,176.8),(0,117.8),(0.6,0.15),(0.59,1.9),---$ & \\
\hline \multirow[t]{3}{*}{ BEBXII } & $0.087,5.007,1.561,31.270,0.318$ & $317.58,325.06,319.80,320.09$ \\
\hline & $(0.077),(3.851),(0.012),(12.940),(0.034)$ & \\
\hline & $(0,0.3),(0,12.6),(1.5,1.6),(5.9,56.6),(0.3,0.4)$ & \\
\hline \multirow[t]{3}{*}{ FBBXII } & $15.194,32.048,0.233,0.581,21.855$ & $317.86,325.34,320.08,320.36$ \\
\hline & (11.58), (9.867), (0.091), (0.067), (35.548) & \\
\hline & $(0,37.8),(12.7,51.4),(0.05,0.4),(0.45,0.7),(0,91.5)$ & \\
\hline \multirow[t]{3}{*}{ FKwBXII } & $14.732,15.285,0.293,0.839,0.034$ & $317.76,325.21,319.98,320.26$ \\
\hline & $(12.390),(18.868),(0.215),(0.854),(0.075)$ & \\
\hline & $(0,39.02),(0,52.27),(0,0.71),(0,2.51),(0,0.18)$ & \\
\hline \multirow[t]{3}{*}{ ZBBXII } & $41.973,---, 0.157,44.263,---$ & $313.86,318.35,314.39,315.36$ \\
\hline & $(38.787),---,(0.082),(47.648),---$ & \\
\hline & $(0,117.99),---,(0,0.32),(0,137.65),---$ & \\
\hline \multirow[t]{3}{*}{ PBXPaII } & $-0.34,0.79,---, 0.19,---$ & 312.3, 316.8, 313.1, 313.8 \\
\hline & $(1.35),(0.266),---,(0.017),---$ & \\
\hline & $(-3.14,2.46),(0.25,1.33),---,(0.15,0.23),---$ & \\
\hline
\end{tabular}


P.P Plot for Breaking Stress of Carbon Fibers

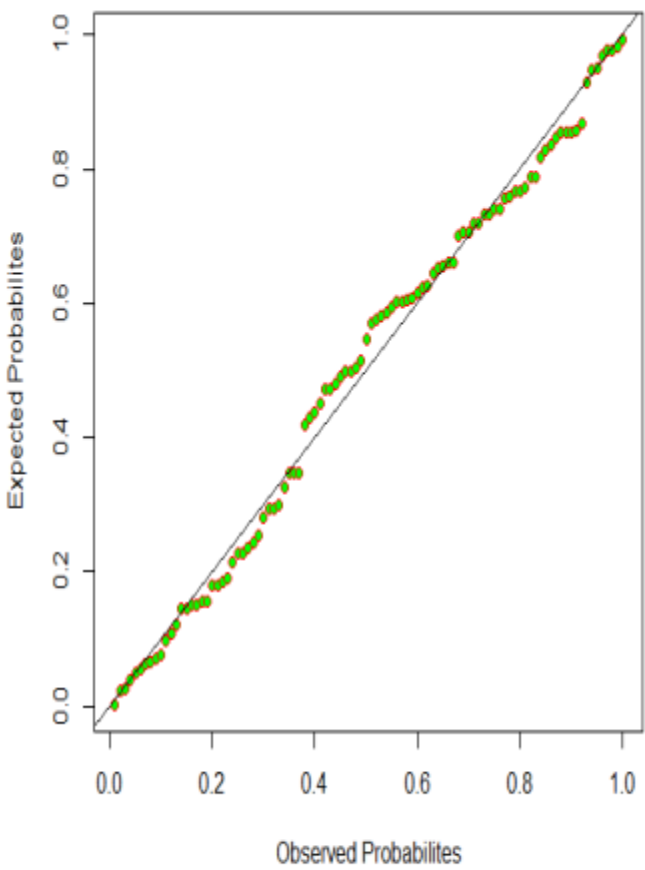

Data set I

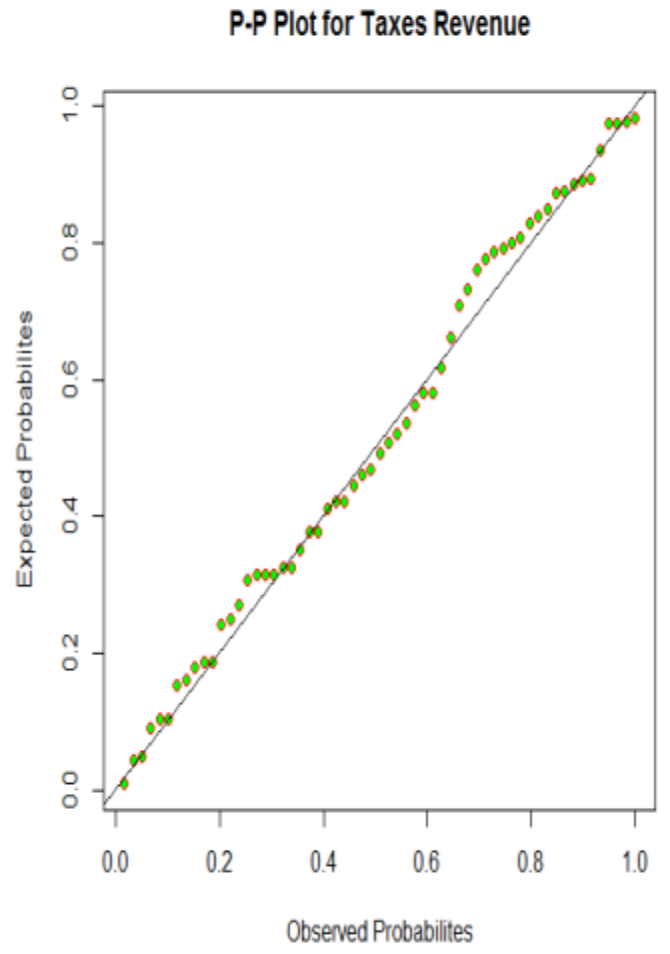

Data set III
P.P Plot for Survival Times

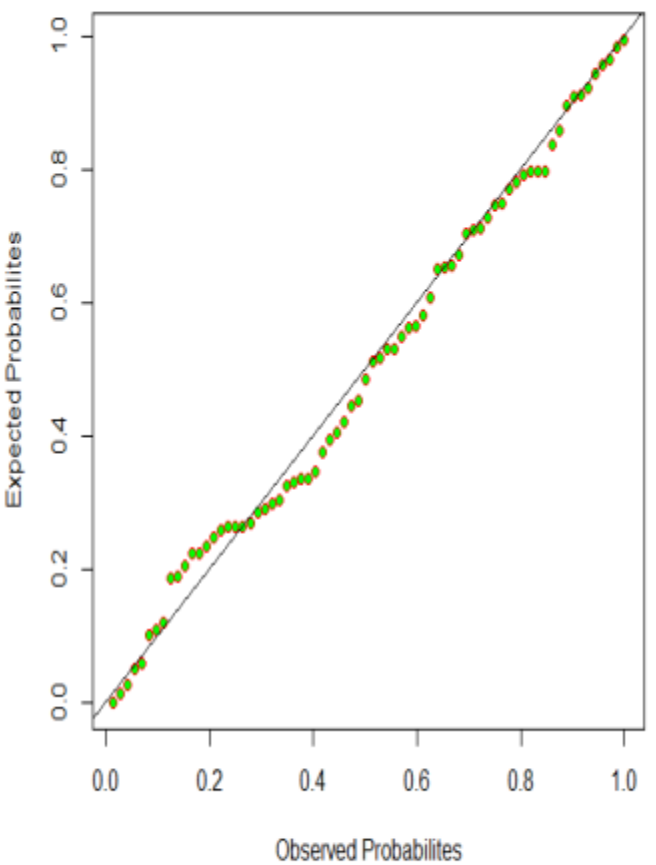

Data set II

PP Plot for Survival Times (Myelogeneous Leukaemia)

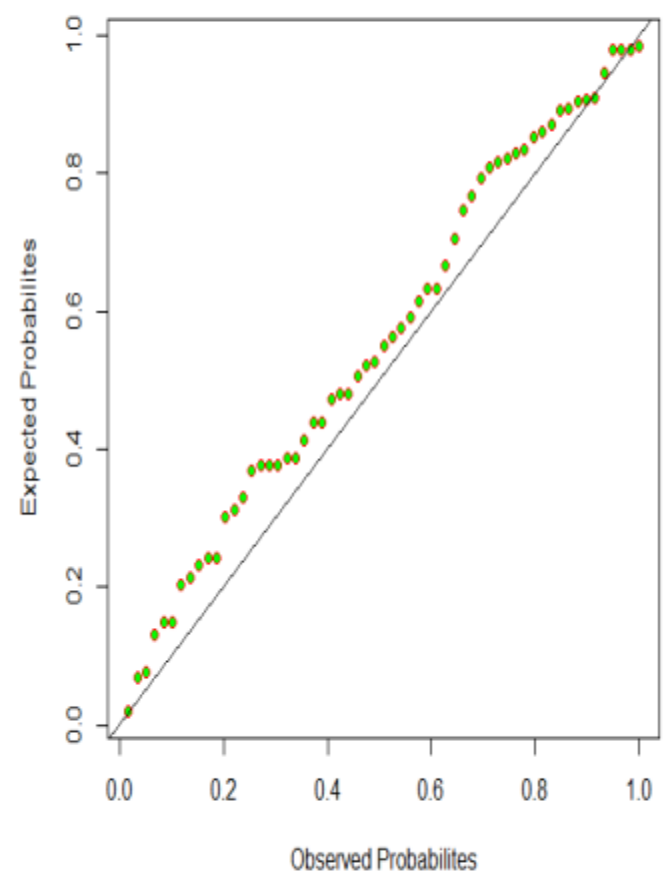

Data set IV

Figure 3: P-P plots.

Pak.j.stat.oper.res. Vol.XV No. III 2019 pp713-730 


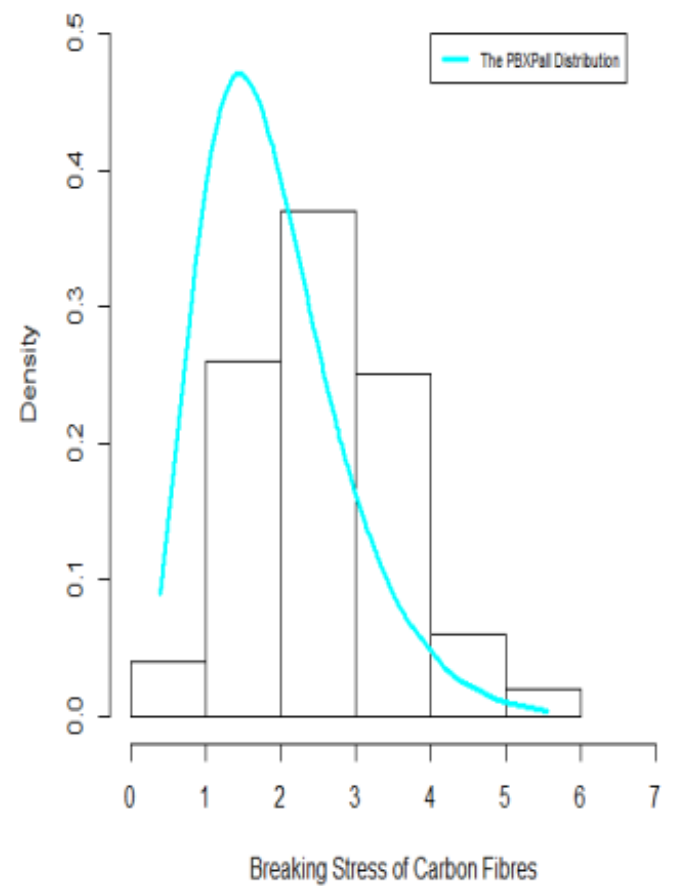

Data set I

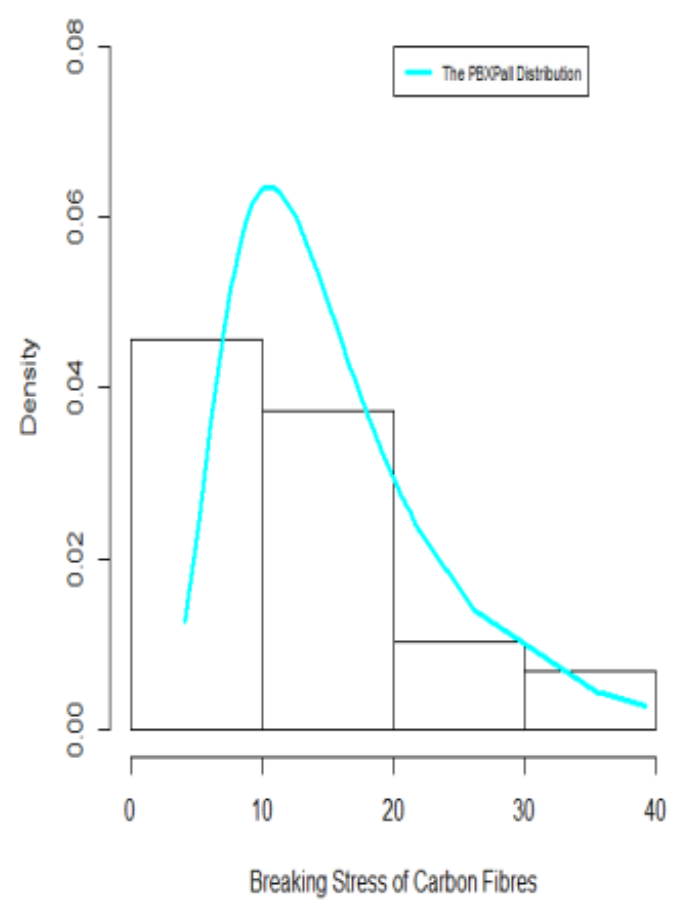

Data set III

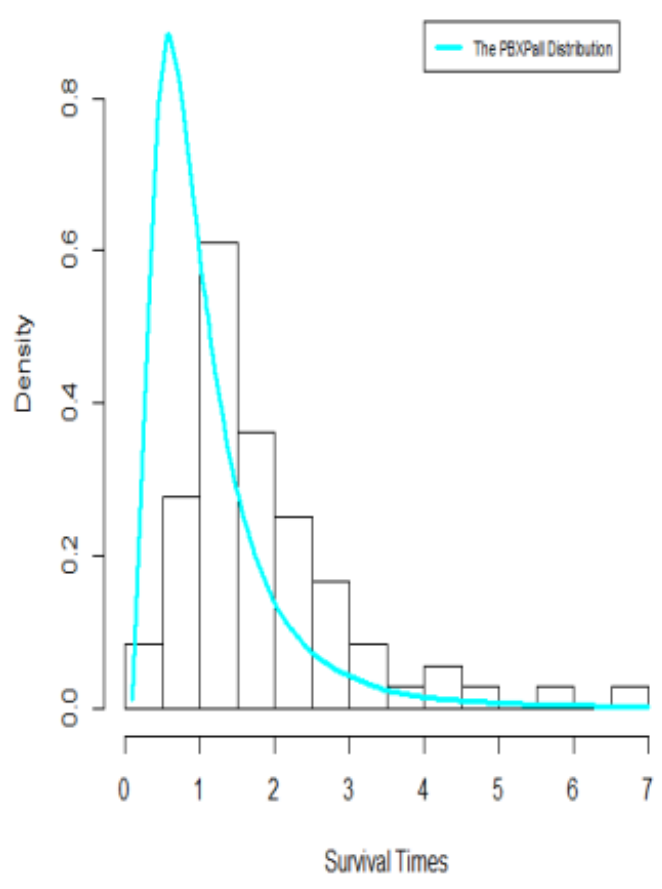

Data set II

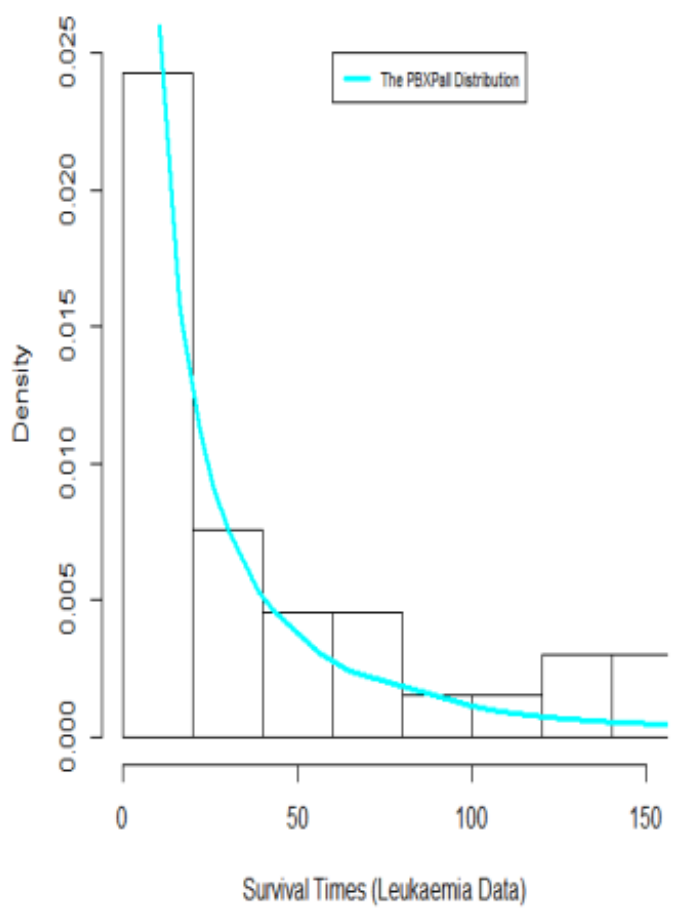

Data set IV

Figure 4: Histograms. 

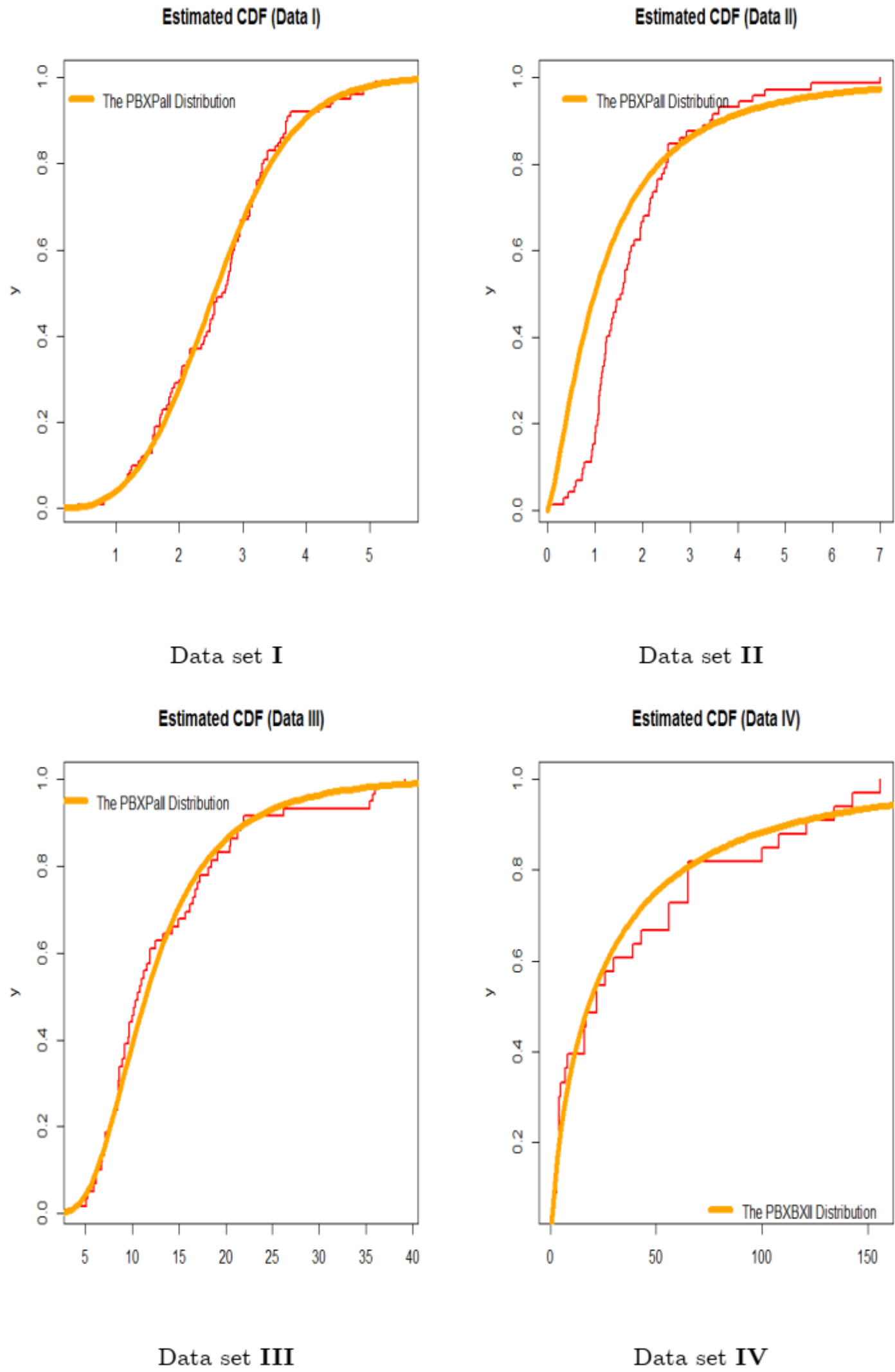

Figure 5: Estimated CDFs.

Pak.j.stat.oper.res. Vol.XV No. III 2019 pp713-730 
Kaplan-Meier Survival Plot

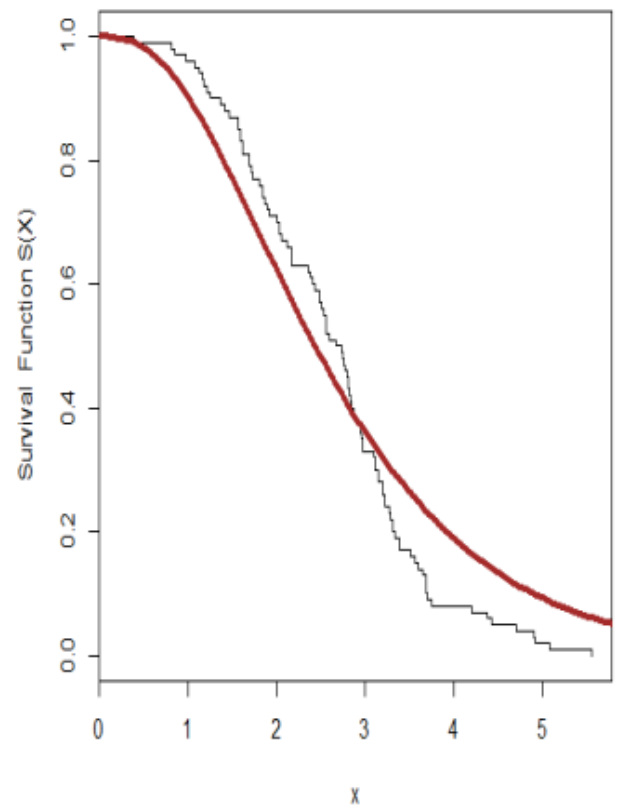

Data set I

Kaplan-Meier Survival Plot

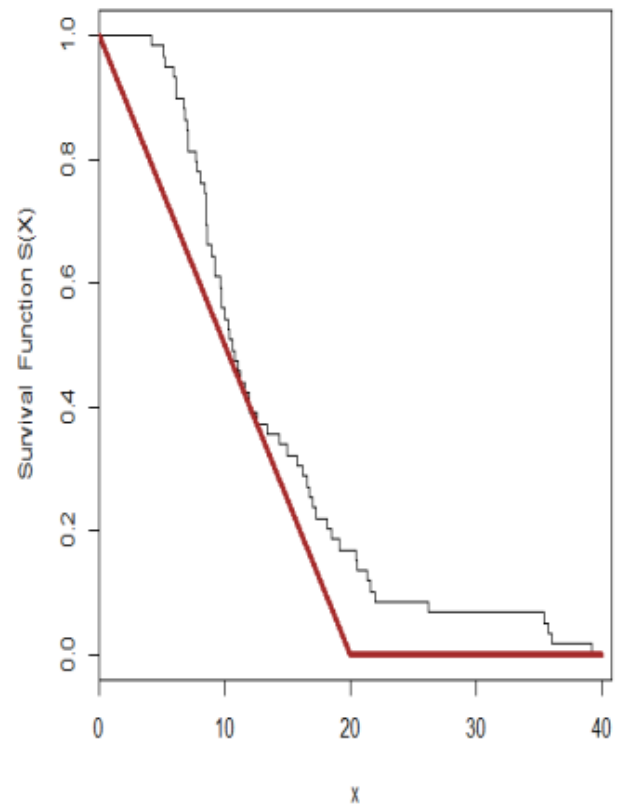

Data set III
Kaplan-Meier Survival Plot

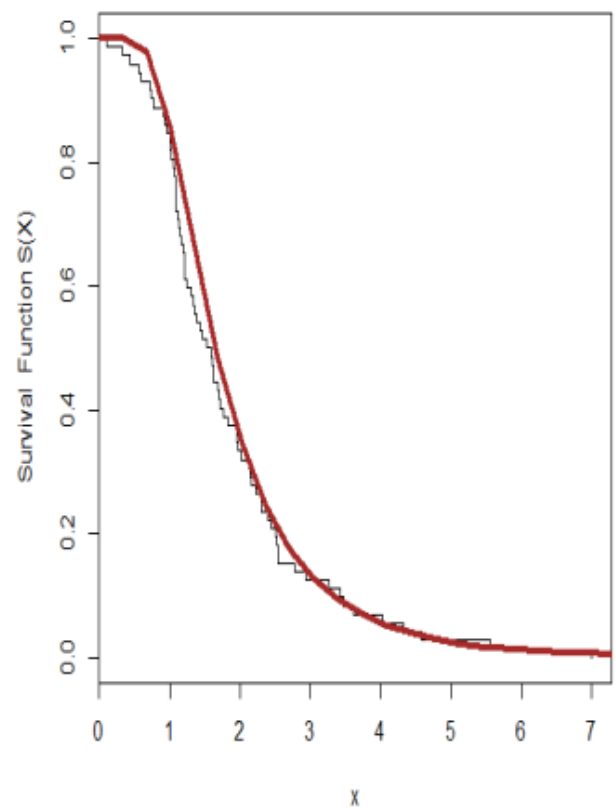

Data set II

Kaplan-Meier Survival Plot

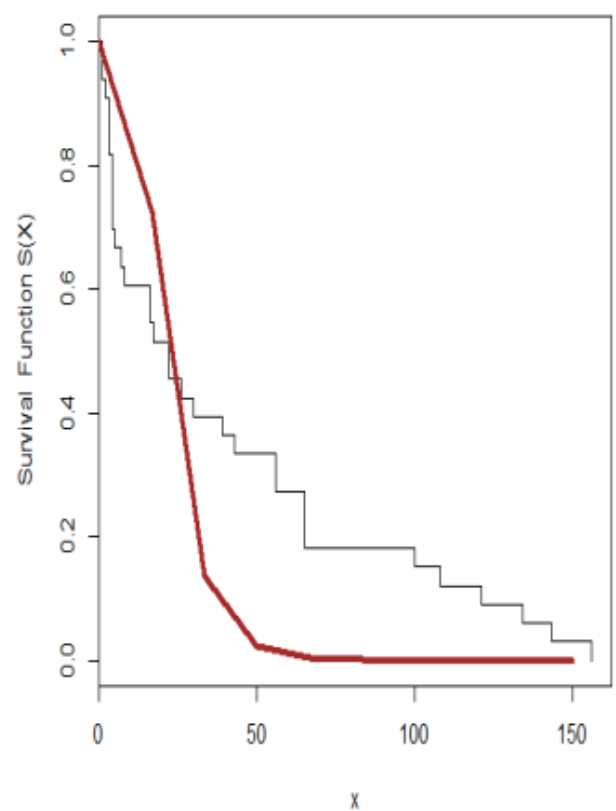

Data set IV

Figure 6: Kaplan-Meier Survival plots. 


\section{Conclusions}

We introduce a new continuous model with strong physical motivations and wide applications upon compounding the discrete zero truncated Poisson model and a new continuous model called the Burr X Pareto type II distribution. Some of its mathematical and statistical properties are derived as well as four applications to real data sets are provided with details to illustrate the wide importance of the new model. We conclude that the new model is better than other nine competitive models as noted via the four applications. Method of maximum likelihood is used to estimate the unknown parameters of the new model. The new model provides adequate fits as compared to other related models in four applications with the smallest values of AIC, BIC, CAIC and HQIC.

\section{References}

1. Aarset, M. V. (1987). How to identify a bathtub hazard rate. IEEE Transactions on Reliability, 36(1), 106-108.

2. Akinsete, A., Famoye, F. and Lee, C. (2008). The beta-Pareto distribution. Statistics, 42, 547-563.

3. Altun, E., Yousof, H. M. and Hamedani G. G. (2018a). A new log-location regression model with influence diagnostics and residual analysis. International Journal of Applied Mathematics and Statistics, forthcoming.

4. Altun, E., Yousof, H. M., Chakraborty, S. and Handique, L. (2018b). ZografosBalakrishnan Burr XII distribution: regression modeling and applications, International Journal of Mathematics and Statistics, forthcoming.

5. Bjerkedal, T. (1960). Acquisition of resistance in Guinea pigs infected with different doses of virulent tubercle bacilli. American Journal of Hygiene, 72, 130-148.

6. Alizadeh, M., Lak, F., Rasekhi, M., Ramires, T. G., Yousof, H. M. and Altun, E. (2018). The odd log-logistic Topp Leone G family of distributions: heteroscedastic regression models and applications. Computational Statistics, forthcoming.

7. Alizadeh, M., Yousof, H. M. Rasekhi, M. and Altun, E. (2019). The odd log-logistic Poisson-G Family of distributions, Journal of Mathematical Extensions, forthcoming.

8. Cordeiro, G. M., Yousof, H. M., Ramires, T. G. and Ortega, E. M. M. (2018). The Burr XII system of densities: properties, regression model and applications. Journal of Statistical Computation and Simulation, 88(3), 432-456.

9. Gomez-Deniz, E. and Calderin-Ojeda, E. (2015). Modelling insurance data with the Pareto ArcTan distribution. ASTIN Bulletin: The Journal of the International, Actuarial Association, 45, 639-660.

10. Bourguignon, M., Silva, M. B., Zea, L. M. and Cordeiro, G. M. (2013). The Kumaraswamy Pareto distribution. Journal of Statistical Theory and Applications 12, 129-144.

11. Gupta, R. C., Gupta, P. I. and Gupta, R. D. (1998). Modeling failure time data by Lehmann alternatives. Communications in Statistics-Theory and Methods 27, 887-904.

12. Hamedani G. G., Altun, E, Korkmaz, M. C., Yousof, H. M. and Butt, N. S. (2018). A new extended $G$ family of continuous distributions with mathematical properties, characterizations and regression modeling. Pak. J. Stat. Oper. Res., 14(3), 737-758.

13. Hamedani G. G. Rasekhi, M., Najibi, S. M., Yousof, H. M. and Alizadeh, M., (2019). Type II general exponential class of distributions. Pak. J. Stat. Oper. Res., forthcoming. 
14. Hamedani G. G. Yousof, H. M., Rasekhi, M., Alizadeh, M., Najibi, S. M. (2017). Type I general exponential class of distributions. Pak. J. Stat. Oper. Res., XIV(1), 39-55.

15. Ibrahim, M. (2019). The compound Poisson Rayleigh Burr XII distribution: properties and applications, Journal of Applied Probability and Statistics, forthcoming.

16. Korkmaz, M. C., Altun, E., Yousof, H. M. and Hamedani G. G. (2019). The Odd Power Lindley Generator of Probability Distributions: Properties, Characterizations and Regression Modeling, International Journal of Statistics and Probability, 8(2), 7089.

17. Korkmaz, M. C., Alizadeh, M., Yousof, H. M. and Butt, N. S. (2018a). The generalized odd Weibull generated family of distributions: statistical properties and applications. Pak. J. Stat. Oper. Res., 14(3), 541-556.

18. Korkmaz, M. C., Yousof, H. M., Hamedani G. G. and Ali, M. M. (2018b). The Marshall-Olkin generalized G Poisson family of distributions, Pakistan Journal of Statistics, 34(3), 251-267.

19. Nadarajah, S. (2005). Exponentiated Pareto distributions. Statistics: A Journal of Theoretical and Applied Statistics, 39(3), 2005, 255-260.

20. Nichols, M.D. and Padgett, W.J. (2006). A bootstrap control chart for Weibull percentiles. Quality and Reliability Engineering International, 22, 141-151.

21. Nascimento, A. D. C., Silva, K. F., Cordeiro, G. M., Alizadeh, M. and Yousof, H. M. (2019). The odd Nadarajah-Haghighi family of distributions: properties and applications. Studia Scientiarum Mathematicarum Hungarica, 56(2), 1-26.

22. Pareto, V. (1896). La courbe de la rpartition de la richesse. In Recueil publi par la Facult de Droit l.occasion de 1.exposition nationale suisse (Eds) C. ViretGenton, Lausanne: Universit de Lausanne, pp. 373-387.

23. Yousof, H. M., Afify, A. Z., Hamedani, G. G. and Aryal, G. (2017a). The Burr X generator of distributions for lifetime data. Journal of Statistical Theory and Applications, 16, 288-305.

24. Yousof, H. M., Altun, E., Ramires, T. G., Alizadeh, M. and Rasekhi, M. (2018a). A new family of distributions with properties, regression models and applications, Journal of Statistics and Management Systems, 21(1), 163-188.

25. Yousof, H. M., Alizadeh, M., Jahanshahiand, S. M. A., Ramires, T. G., Ghosh, I. and Hamedani G. G. (2017b). The transmuted Topp-Leone G family of distributions: theory, characterizations and applications, Journal of Data Science. 15, 723-740.

26. Yousof, H. M., Majumder, M., Jahanshahi, S. M. A., Ali, M. M. and Hamedani G. G. (2018b). A new Weibull class of distributions: theory, characterizations and applications, Journal of Statistical Research of Iran, 15, 45-83.

27. Yousof, H. M., Rasekhi, M., Altun, E., Alizadeh, M. Hamedani G. G. and Ali M. M. (2019). A new lifetime model with Bayesian estimation, characterizations and applications. Communications in Statistics-Simulation and Computation, 48(1), 264286. 